ORIGINAL

\title{
Obtención y evaluación del semen de capibara Hydrochoerus hydrochaeris
}

\section{Collection and evaluation of semen from the Capybara Hydrochoerus hydrochaeris}

\author{
José Rodríguez $\mathrm{P}^{1}{ }^{1}$ M.Sc, Miguel Peña J, ${ }^{1}$ MVZ, Agustín Góngora O, ${ }^{1 *}$ Ph.D, \\ Ricardo Murillo $\mathrm{P}^{1}{ }^{1} \mathrm{MVZ}$.
}

\begin{abstract}
${ }^{1}$ Universidad de los Llanos, Escuela de Ciencias Animales. Grupo de Investigación en Reproducción y Genética Animal, (GIRGA). Kilómetro 12 vía Puerto López, Villavicencio-Meta, Colombia. *Correspondencia: agongora@unillanos.edu.co
\end{abstract}

Recibido: Julio de 2011; Aceptado: Marzo de 2012.

\section{RESUMEN}

Objetivo. Determinar las características del semen y la morfometría de los espermatozoides del Capibara (Hydrochoerus hydrochaeris). Materiales y métodos. Se utilizaron 10 machos con peso entre $21-45 \mathrm{~kg}$, los cuales fueron restringidos y anestesiados. El semen se obtuvo mediante electroeyaculación y se determinó el color, volumen, $\mathrm{pH}$, motilidad en masa, motilidad individual, viabilidad, concentración y morfología. Se realizaron además mediciones de la cabeza y la cola de los espermatozoides. Resultados. Se obtuvo semen en el 100\% (10/10) de los animales. El mayor número de eyaculaciones $(80 \% ; 8 / 10)$, se obtuvo con un voltaje máximo de $6 \mathrm{~V}$. El color fue blanco, de aspecto lechoso, los valores promedio fueron volumen $135.5 \pm 93.56 \mu \mathrm{l}, \mathrm{pH} 8.14 \pm 0.38$, motilidad masal $32.60 \pm 13.46 \%$, motilidad individual $34 \pm 19.81 \%$, viabilidad $51.3 \pm 19.42 \%$, concentración espermática $127 \pm 59.01 \times 106$ espermatozoides $/ \mathrm{mL}$ y morfología $51.3 \pm 19.42$ espermatozoides normales. La longitud de la cabeza fue $5.41 \pm 0.7 \mu \mathrm{m}$, el ancho de la cabeza $3.77 \pm 0.5 \mu \mathrm{m}$ y área de la cabeza $75.66 \pm 20.6 \mu \mathrm{m}^{2}$. La longitud de la cola fue $27.9 \pm 11.3 \mu \mathrm{m}$. Conclusiones. La obtención del semen fue satisfactoria mediante electroeyaculación, sin presentar notables diferencias en las características del semen y morfología de los espermatozoides con otros roedores silvestres de menor tamaño, aunque se observó una alta variabilidad de estas características entre los animales muestreados posiblemente por la heterogeneidad de los animales experimentales.

Palabras clave: concentración espermática, motilidad individual, motilidad masal (Fuente: AIMS).

\section{ABSTRACT}

Objetive. Determine the characteristics of semen and morphometry of the Capybara (Hydrochoerus hydrochaeris) spermatozoid. Materials and methods. 10 males, weighing between $21-45 \mathrm{~kg}$, which were restrained and anesthetized, were used in the study. The semen sample was obtained by electroejaculation and the color, volume, $\mathrm{pH}$, mass motility, individual motility, viability, concentration and morphology were determined. Measurements of the head and tail of spermatozoids were also 
conducted. Results. Semen was obtained from $100 \%(10 / 10)$ of the animals. The highest number of ejaculations $(80 \% ; 8 / 10)$ was obtained with a maximum voltage of $6 \mathrm{~V}$. The color was white, of a milky appearance, average values were volume $135.5 \pm 93.56 \mu \mathrm{l}, \mathrm{pH} 8.14 \pm 0.38$, mass motility $32.60 \pm 13.46 \%$, individual motility $34 \pm 19.81 \%$, viability $19.42 \pm 51.3 \%$, sperm concentration 127 $\pm 59.01 \times 106$ spermatozoids / $\mathrm{mL}$ and morphology $51.3 \pm 19.42$ normal spermatozoids. The head length was $5.41 \pm 0.7 \mu \mathrm{m}$, the width of head $3.77 \pm 0.5$ and head area $75.66 \pm 20.6 \mu \mathrm{m}^{2}$. The tail length was of $27.9 \pm 11.3 \mu \mathrm{m}$. Conclusions. Semen collection by electro ejaculation was successful, without presenting significant differences in semen characteristics and spermatozoid morphology with other smaller wild rodents, although there was a high variability of these characteristics observed between the animals sampled, possibly due to the heterogeneity of the experimental animals.

Key words: Gross motility, individual motility, sperm concentration (Source: AIMS).

\section{INTRODUCCIÓN}

Las especies silvestres se han constituido, con cierta reserva, en una alternativa de explotación zootécnica con el fin de suplir las demandas de proteína animal de la humanidad, además han hecho parte de la cultura ancestral de los pueblos (1). Se tienen evidencias que desde la época precolombina, hacían parte esencial de la dieta de las comunidades indígenas (2). El capibara o chigüiro es un mamífero de alto valor biológico que hace parte de este grupo de especies, es considerado el mayor roedor del mundo y habita en las sabanas y áreas inundables de varios países de Centro y Suramérica (3).

Por la calidad de la carne y la piel, el capibara es cazado por los pobladores locales a partir de las poblaciones naturales, aunque esta situación viene cambiando, dado que su fácil domesticación permite que se explote comercialmente en Argentina (4), Brasil $(1,5)$ Colombia (6) y Venezuela (7).

En Colombia las mayores poblaciones de chigüiro se localizan en la región de los Llanos Orientales, especialmente en las sabanas de los departamentos de Arauca, Meta, Casanare y Vichada (6). La población no se encuentra amenazada, sin embargo, ciertos factores antrópicos como la caza indiscriminada y los cambios en los sistemas de producción animal plantean un futuro incierto para su sobrevivencia.

Del capibara se conocen aspectos relacionados con la biometría corporal y características intestinales (8), comportamiento social (9-11) hábitos alimenticios (12), pubertad (13) y ciclo estral (14), sin embargo, no hay información sobre las características seminales.

El objetivo de este estudio fue evaluar las características del semen y determinar el tamaño de los espermatozoides del capibara, como paso previo a su crioconservación y el establecimiento de bancos de germoplasma para posteriores desarrollos investigativos y biotecnológicos.

\section{MATERIALES Y MÉTODOS}

Sitio de estudio y animales. Se utilizaron 10 machos adultos con un rango de peso entre 21-45 kg, sexualmente maduros, mayores de 15 meses, provenientes de dos parques agroecológicos de la ciudad de Villavicencio, departamento del Meta (temperatura media de $27^{\circ} \mathrm{C}$ y humedad relativa del $77 \%$ ). Los animales permanecían en espacios abiertos con hembras en grupo no mayores de 10 animales, antes de la toma de semen fueron mantenidos en ayuno por 12 horas. Todos los procedimientos utilizados fueron aprobados por el comité de bioética de la Universidad de los Llanos, acta No 026 de Abril de 2010.

Anestesia. Se realizó restricción física de los animales con una malla de Nylon de calibre No 4 y ojo de malla de $5 \mathrm{~cm}$, pre medicados con atropina $0.044 \mathrm{mg} / \mathrm{kg} \mathrm{IM} \mathrm{(Atropina} \mathrm{zoo}{ }^{\circledR}$ ), luego de 10 minutos se sedaron con ketamina $5 \mathrm{mg} /$ kg IM (Ketamina $50^{\circledR}$, Holliday ${ }^{\circledR}$ ) mas xilacina $0.2 \mathrm{mg} / \mathrm{kg}$ IM (Xilacina 20 ${ }^{\circledR}$, Holliday ${ }^{\circledR}$ ) de acuerdo con la técnica descrita por West et al (15). La dosis de inducción no superó el 50\% de la dosis total con el fin de mantener un plano de anestesia superficial. Durante todo el tiempo que duró el procedimiento se monitorearon las constantes fisiológicas como temperatura, frecuencias cardiaca y respiratoria.

Obtención del semen. Cada animal fue suspendido en la misma malla con que había sido restringido y colocado en posición de decúbito esternal, se realizó evacuación de las heces del recto mediante la introducción de 1 o 2 dedos con la mano enguantada, y luego se procedió al lavado del prepucio con suficiente agua y jabón. Se secó con papel absorbente toda la región desde el ano hasta el prepucio. Se introdujo una sonda transrectal de $2.5 \mathrm{~cm}$ de diámetro $x 16 \mathrm{~cm}$ de longitud previamente lubricada y acoplada a un electroeyaculador (Electrojac 5 Ideal Instruments ${ }^{\circledR}$ Michigan-USA). La electro estimulación se realizó de forma manual iniciando con 1 Voltio por 4 segundos, seguido 
por 4 segundos de descanso y paulatinamente aumentando el voltaje a razón de 1 voltio hasta 6 voltios cada uno con su respectivo periodo de descanso hasta la obtención del semen. La colección del semen se realizó en tubos cónicos plásticos graduados de $1.5 \mathrm{~mL}$ (Flex Tubes ${ }^{\circledR}$ Eppendorf, New York, Estados Unidos).

Evaluación del semen. Obtenido el semen se determinó el color contrastando la muestra sobre un fondo oscuro, el volumen total de la muestra se midió con una micropipeta graduada de 5 a $200 \mu \mathrm{l}$ (Eppendorf Research ${ }^{\circledR}$ New York, Estados Unidos). Se determinó el pH impregnando una tira de papel indicador (Universal Indicator ${ }^{\circledR}$ Merck, Darmstadt, Alemania) con $20 \mu \mathrm{l}$ de la muestra. La motilidad en masa se obtuvo colocando $10 \mu$ l en una lámina portaobjetos temperada a $35^{\circ} \mathrm{C}$ sobre una platina térmica (HT50 ${ }^{\circledR}$ Minitube, Tiefenbach, Alemania) y observación directa en un microscopio de luz a $10 x$ (CX31 ${ }^{\circledR}$ Olympus, Tokyo, Japon). La motilidad individual se observó directamente. Para determinar la viabilidad y morfología se mezclaron $20 \mu \mathrm{l}$ de la muestra con $20 \mu \mathrm{l}$ de eosina nigrosina al 2\% (Certistain ${ }^{\circledR}$ Merck, Darmstadt, Alemania) y se realizó un extendido fino dejando secar las láminas por $30 \mathrm{~min}$. Los frotis se observaron en microscopio óptico (CX31 ${ }^{\circledR}$ Olympus, Tokyo, Japón) con objetivo de inmersión, se contaron 200 espermatozoides por placa. Los resultados se expresaron en porcentaje de espermatozoides vivos. La concentración se determinó diluyendo $10 \mu \mathrm{l}$ de semen en $1.0 \mathrm{~mL}$ de solución salina formolada al $10 \%$ y se realizó el conteo en cámara de Neubauer ${ }^{\circledR}$. Los resultados se expresaron en millones/mL.

Morfometría de los espermatozoides. Se utilizó el software Image J, un sistema semiautomático de procesamiento y análisis de imágenes (16) de acuerdo con la metodología propuesta por Cruz (17), se seleccionaron al azar 42 espermatozoides de los frotis utilizados para valorar la morfología. Se tomó un cuadrado con la región de interés (ROI por sus siglas en inglés) en donde estuviese la cabeza del espermatozoide. Se procedió a descomponer en las componentes de color (rojo, verde y azul) de la representación original de la imagen para seleccionar la componente azul donde se presentaba un mejor contraste entre las cabezas de los espermatozoides y el fondo. Se realizó un proceso de mejoramiento de contraste y brillo para resaltar la región de la cabeza de los espermatozoides, seguidamente se segmentó la región asociada a la cabeza utilizando el método de crecimiento de regiones, el cual consiste en elegir a una serie de píxeles iniciales, denominados puntos semilla; a los que se añaden nuevos píxeles con propiedades similares para constituir una región. Para ello, se examina si los píxeles contiguos a las semillas tienen niveles de gris parecidos, si es así, se asume que pertenecen a la misma región y pasan a tener los mismos valores que los puntos iniciales. Es decir, si se cumple que la diferencia entre el nivel de gris $f(x, y)$ de un píxel en la imagen y el nivel de gris del píxel del punto semilla $f\left(S_{x i} S_{y i}\right)$ es menor que un valor de umbral preestablecido $T$, entonces el pixel de las coordenadas $(\mathrm{x}, \mathrm{y})$ es asociado a la región tal como se describe en la siguiente ecuación (18).

$$
\left|f(x, y)-f\left(s_{x i}, s_{y i}\right)\right|<T
$$

Finalmente la región seleccionada por el crecimiento de regiones (Figura 1) fue binarizada (Figura 2), lo que significa crear una nueva imagen que tendrá el valor de uno en la región asociada a la cabeza y cero en el resto de la imagen, esto para separar el objeto de interés (la cabeza del espermatozoide) para luego aplicar las medidas morfológicas. Antes de aplicar la descripción morfométrica de la cabeza, se aplicó la operación de morfología matemática conocida como cierre para depurar, suavizar y definir mejor el contorno de la forma de la cabeza.

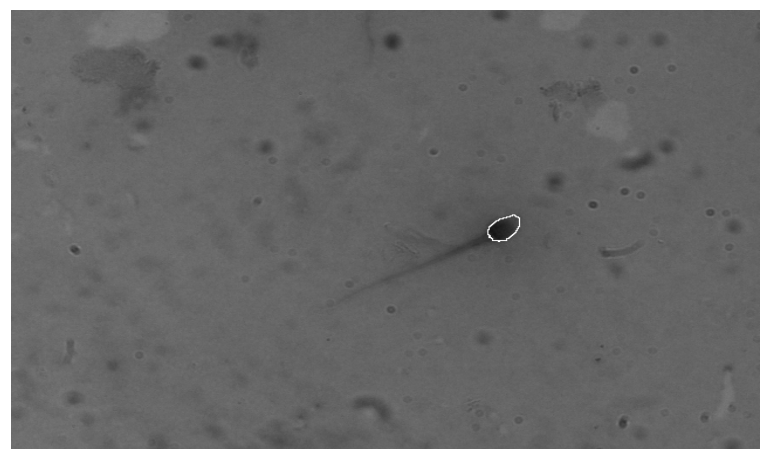

Figura 1. Región seleccionada de la cabeza del espermatozoide por el método de crecimiento de regiones.

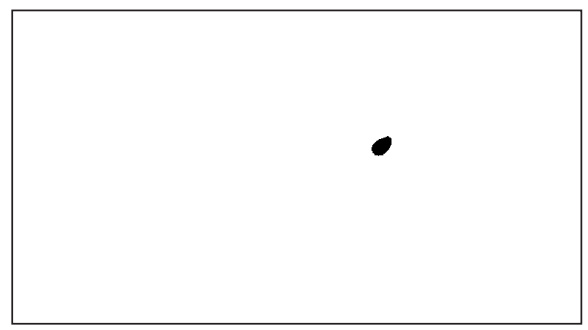

Figura 2. Imagen binarizada de la cabeza del espermatozoide proveniente de la figura 1. 
Descriptores morfológicos. En el análisis morfométrico se utilizaron algunos descriptores de forma usados convencionalmente en el procesamiento de imágenes, que fueron provistos por el software Image J para el análisis de partículas fijando el tipo de medidas. La descripción de cada uno de los descriptores morfológicos utilizados en este estudio fue:

Área (A). Número de píxeles dentro de la cabeza, incluyendo los bordes.

$$
A=\sum_{i=0}^{N-1} \sum_{j=0}^{N-1} g(i, j)
$$

Perímetro (P). Número de píxeles que forman el borde de la cabeza.

Compacidad. Combinación de los dos anteriores e invariante al tamaño del objeto. También llamado circularidad ya que alcanza su mínimo valor de $4 \pi$ para un círculo.

$$
\frac{P^{2}}{A}
$$

Largo (L). Es la longitud mayor de la cabeza del espermatozoide, incluyendo el acrosoma. En la figura 3 se ilustra con la línea amarilla.

Ancho (W). Es la longitud menor de la cabeza que es perpendicular al largo (L). En la figura 3 se ilustra con una línea verde.

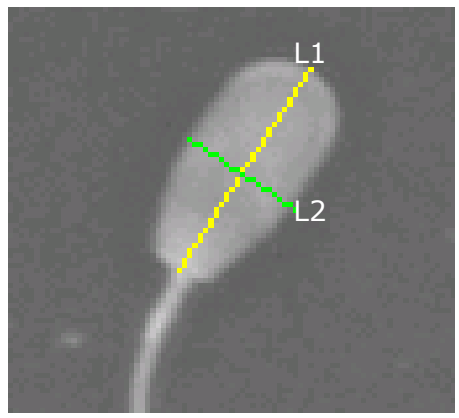

Figura 3. Largo (Línea amarilla L1) y ancho (Línea verde L2) de la cabeza del espermatozoide.

Circularidad. Esta medida de forma describe el grado de circularidad que tiene el objeto de interés. De tal forma que tiene un valor de 1 cuando es un círculo perfecto y 0 cuando es una línea y está definida por la siguiente fórmula:

$$
\frac{P^{2}}{4 \pi \times A}
$$

Excentricidad. También conocido como relación de aspecto o factor de forma. Está definido como la razón entre el largo y el ancho.
Redondez. Es un factor de forma cuyo mínimo valor es la unidad correspondiente a un círculo. Está definido por la siguiente formula, en donde el factor de ajuste 1.064 corrige el perímetro debido al efecto de bordes producido por la digitalización de la imagen.

$$
\frac{P^{2}}{4 \pi \times A \times 1.064}
$$

Elongación. Está definida como la razón entre la diferencia y la suma del largo y ancho, tal como se presenta en la siguiente fórmula:

$$
(L-W) /(L+W)
$$

Medidas de la cola del espermatozoide. Se obtuvieron dos medidas, la longitud total de la cola desde su inserción hasta el final de la pieza terminal y la distancia de extremo a extremo de ésta. En la figura 4 se presenta una imagen de ejemplo a la cual se le realizaron éstas medidas, en rojo se ilustra el marcado de la longitud de la cola (LC) y en amarillo la distancia de extremo a extremo (DEE). Una relación entre estas dos medidas permite ver el grado de torsión de la cola, lo cual determina los tipos más comunes de anormalidades de la cola.

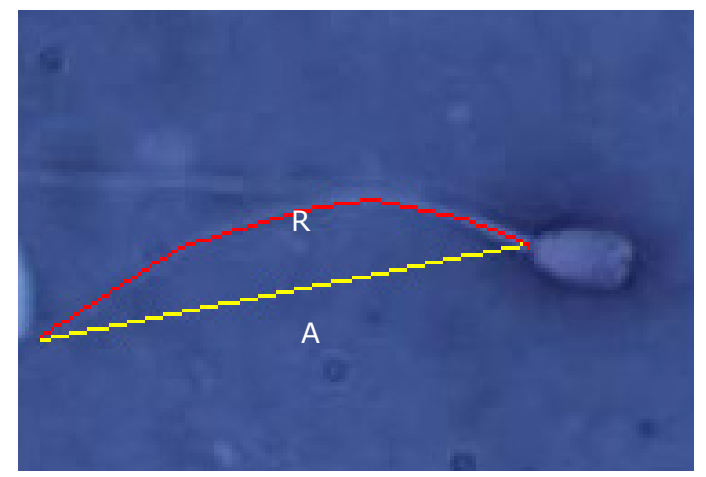

Figura 4. Comparación de las medidas de la cola. Longitud de la cola (rojo R) y distancia entre extremos de la cola (amarillo A).

Análisis estadístico. Se creó una base de datos en Microsoft Excel,2010 y los resultados analizados mediante estadística descriptiva con el programa Statistical Package for Social Sciences SPSS 17.0. Los datos se expresaron en valores promedio, desviación estándar, máximos y mínimos.

\section{RESULTADOS}

No se observó efectos adversos de la anestesia sobre las constantes fisiológicas. La respuesta a la electroeyaculación fue del $100 \%(10 / 10)$ y en el $80 \%$ de las muestras (8/10) se observaron 
espermatozoides. El voltaje en el cual se obtuvo la muestra fue de $6 \mathrm{~V}$ en 8 de 10 animales. El eyaculado fue de color blanco lechoso, las demás características del semen se presentan en la tabla 1.

Tabla 1. Características del semen de capibara obtenido mediante electroeyaculación $(n=10)$.

\begin{tabular}{lc}
\hline Características del semen del capibara & Media \pm SD \\
\hline Volumen $(\mu \mathrm{L})$ & $135.5 \pm 93.56$ \\
$\mathrm{pH}$ & $8.14 \pm 0.38$ \\
Motilidad masal $(\%)$ & $32.60 \pm 13.46$ \\
Motilidad individual (\%) & $34 \pm 19.81$ \\
Viabilidad (\%) Espermatozoides vivos & $51.3 \pm 19.42$ \\
Concentración (Espermatozoides x 106/ml) & $127 \pm 59.01$ \\
Morfología (\%) Espermatozoides normales & $51.3 \pm 19.42$ \\
\hline
\end{tabular}

Tabla 2. Mediciones de la cabeza y la cola de espermatozoides de capibara obtenidas mediante el software Image $\mathrm{J}$.

\begin{tabular}{cccc}
\hline & Media \pm SD & Máximo & Mínimo \\
\hline Cabeza & & & \\
\hline $\mathrm{L}(\mu \mathrm{m})$ & $5.41 \pm 0.7$ & 7.37 & 3.76 \\
$\mathrm{~A}(\mu \mathrm{m})$ & $3.77 \pm 0.5$ & 5.70 & 2.54 \\
$\operatorname{Ar}(\mu \mathrm{m} 2)$ & $75.66 \pm 20.6$ & 46.22 & 153.51 \\
$\mathrm{P}(\mu \mathrm{m})$ & $15.27 \pm 2.2$ & 23.62 & 11.81 \\
$\mathrm{C}(\mu \mathrm{m})$ & $0.86 \pm 0.05$ & 0.95 & 0.74 \\
$\mathrm{E}(\mu \mathrm{m})$ & $1.39 \pm 0.2$ & 1.72 & 0.00 \\
$\mathrm{R}(\mu \mathrm{m})$ & $0.69 \pm 0.14$ & 0.92 & 0.00 \\
$\mathrm{EI}(\mu \mathrm{m})$ & $0.17 \pm 0.03$ & 0.32 & 0.03 \\
Cola & & & \\
\hline $\mathrm{LC}(\mu \mathrm{m})$ & $27.9 \pm 11.3$ & 40.5 & 0.00 \\
$\mathrm{DEE}(\mu \mathrm{m})$ & $25.05 \pm 10.7$ & 0.37 & 0.00 \\
\hline
\end{tabular}

L:largo; A:ancho; Ar:área; P:perímetro; C:circularidad; E: excentricidad; R:redondez; El:elongación; LC:longitud de la cola; DEE:distancia de extremo a extremo.

Las principales alteraciones morfológicas de los espermatozoides fueron cabezas en punta de diamante $24 \pm 9.67(19.7 \%)$, cabezas redondas $9.37 \pm 8.37(7.7 \%)$ y cabezas alargadas $3.25 \pm 6.11(2.7 \%)$. Las medidas de la cabeza y la cola de los espermatozoides se presentan en la tabla 2.

\section{DISCUSIÓN}

El protocolo anestésico utilizado no afectó las constantes fisiológicas, ni puso en riesgo la vida de los animales, por lo que se considera una técnica segura y sugiere su utilización en otros mamíferos silvestres de menor tamaño. Sin embargo, se requieren estudios que permitan evaluar otros protocolos anestésicos, como el propofol, que ya fue utilizado en el pecarí de collar rojo (Tayassu tajacu), donde se obtuvo un mayor volumen de semen mediante electroeyaculación (19).

La obtención del semen en el capibara fue posible mediante electroeyaculación y constituye el primer reporte en esta especie. No existen anteriores estudios que permitan contrastar estos resultados, por lo que pueden ser tomados como valores de referencia. Mediante la misma técnica se ha obtenido semen en mamíferos roedores de menor tamaño como agouti (Dasyprocta leporina) (20) y el pecarí de collar rojo (Tayassu tajacu) (19). En otros estudios mediante la electroeyaculación en roedores y cerdos de guinea, los resultados de las características seminales han sido variables (21).

Los valores del volumen y la concentración del semen deben ser analizados con cuidado, ya que no representan valores reales. Es conocido que mediante electroeyaculación se obtiene muestras más diluidas por una mayor secreción de las glándulas anexas. La concentración espermática (127 x 106 espermatozoides/mL) fue menor a la observada en Agoutí (Dasyprocta agutí) (748 x 106 espermatozoides $/ \mathrm{mL})$. Igual motivo de discusión plantea la obtención de las muestras de semen bajo anestesia ya que se ha encontrado un mayor porcentaje de éxito en animales conscientes versus animales anestesiados $(22,23)$.

$\mathrm{El} \mathrm{pH}$ básico del semen $(\mathrm{pH}:$ 8.14) fue menor aunque sin grandes diferencias al encontrado en agouti (Dasyprocta leporina)( $\mathrm{pH}$ : 8.3) (19) y difiere notablemente del reportado en espermatozoides de epidídimo en Agouti (Dasyprocta agutí) (pH: 6.9) (24), lo que sugiere que la característica básica del semen proviene en gran parte de las glándulas anexas.

El alto porcentaje de anormalidades espermáticas puede estar asociado con los animales muestreados, ya que no se seleccionaron de acuerdo con su organización social (machos dominantes y subordinados), es posible que aquellos subordinados tuviesen un menor número de montas y que los espermatozoides llevaran un mayor tiempo de retención en la cola del epidídimo que conllevara a alteraciones morfológicas. Otro factor posiblemente implicado podría estar asociado con la época en que se realizó la toma de semen (por fuera de la época reproductiva). Si bien el capibara puede aparearse durante todo el año, bajo condiciones adecuadas de cautiverio, presentan dos ciclos de apareamiento especialmente a la entrada de la época de lluvias (6). 
La baja concentración espermática comparada con otros roedores de menor tamaño puede ser el resultado de la técnica utilizada o el menor índice somático gonadal encontrado en estudios histológicos (25). Moreira et al (26), demostraron que el testículo del capibara está constituido de una mayor cantidad de tejido intersticial (células de Leidy) que de tejido productor de espermatozoides (túbulos seminíferos).

Respecto a la morfometría de los espermatozoides, diversos sistemas automáticos o semiautomáticos han sido utilizados para su determinación. Las medidas de la longitud y ancho de la cabeza de los espermatozoides del capibara ( $L: 5.41 \pm 0.7$; $A: 3.77 \pm 0.5)$ fueron similares a los obtenidos en

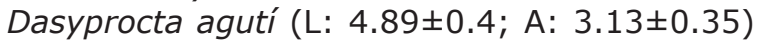
(24). En este estudio fue evidente a la vez, una alta variabilidad en el tamaño de los espermatozoides lo que coincide con estudios en otras especies animales en las cuales dicho hallazgo se ha asociado con la presencia de diferentes subpoblaciones de espermatozoides (27), aunque esto todavía no ha sido reportado en el capibara. Igualmente podría estar asociado con la variación en la morfología relacionada con la edad de los animales la cual fue altamente variable y ha sido encontrada en cerdos (28). La longitud de la cola de los espermatozoides
$(27.9 \pm 1.1 \mu \mathrm{m})$ fue menor al reportado en un estudio que incluyó 445 especies de mamíferos incluido el capibara (29). Sin embargo, es similar a la longitud reportada en agutí (Dasyprocta agutí) (29.91 $\pm 2.29 \mu \mathrm{m})(24)$.

Se concluye que la obtención de semen en el capibara mediante electroeyaculación es posible y que las características del semen y morfométricas de los espermatozoides son similares a las reportadas en otros roedores de menor tamaño. La obtención de semen y evaluación de sus características en esta especie, determina la posibilidad de su crio conservación y su aplicación con fines biotecnológicos o investigativos.

\section{Agradecimientos}

Universidad de los Llanos por la financiación de este proyecto. Profesionales y personal administrativo del parque los Ocarros y Merecure. Licenciada María Rocío Parra auxiliar del laboratorio de Reproducción y Genética Animal de la Universidad de los Llanos. Estudiantes Mónica Tatiana Rincón y Roger Alexis Morales, Ingeniero Ángel Cruz por la digitalización de las imágenes y medición de los espermatozoides.

\section{REFERENCIAS}

1. Nogueira Filho, SLG. Manual de Criação da Capivara. Viçosa-MG, Brasil: Centro de produções técnicas - CPT; 1996.

2. Humbolt A. Von. Voyage aux régions équinoxiales du Nouveau Continent fait en 1799, 1800, 1801, 1802, 1803 et 1804, par Al. de Humbolt et A. Bompland. 1. Relation historique, N. Maze, Paris, 1819; 2: 1-722.

3. Ojasti J. Human explotation of capybara. In: Neotropical Wild life Use and Conservation. Ed: JG Robinson and KH Redford. USA: The University of Chicago Press: 1991.

4. Alvarez MR. Manejo sustentable del carpincho (Hydrochoerus hydrochaeris, Linnaeus 1766) en Argentina: un aporte al conocimiento de la biología de la especie desde la cría en cautiverio [Tesis Doctoral]. Buenos Aires, Argentina: Universidad de Buenos Aires; 2002.
5. Hosken F M. Criação e manejo de capivaras, Cuiabá/MT, Brazil: Edição Sebrae; 1999.

6. Fuerbringer J. The capybara: a practical manual for raising them in captivity in Colombia, Temas Orientación Agropecuaria 1974; 99:5-59.

7. Parra R, Escobar A, González-Jiménez E. El chigüire: su potencial biológico y su cría en confinamiento, Informe Anual del Instituto de Producción Animal, Maracay, Venezuela: Universidad Central de Venezuela; 1978.

8. Toshio OL, Soares BM, Fonseca CC, Rego de Paula TA, Neves MT. Aspectos biométricos y corporais e dos intestinos da capivara Hydrochoerus hydrochaeris, com em fases no desenvolvimento do ceco. Biotemas 2004; 17:177-190.

9. Azcárate T. Sociobiología y manejo del capibara (Hydrochoerus hydrochaeris), Doñana Acta Vertebrata 1980; 7:1-228. 
10. Herrera EA, MacDonald D. Group stability and the structure of a capybara population. Symp Zool Soc Lond 1987; 58:115-130.

11. Maldonado-Chaparro A, Blumstein DT. Management implications of capibara (Hydrochoerus hydrochaeris) social behavior. Biological Conservation 2008; 141:1945-1952.

12. Silva Neto PB. Alimentação e manejo de Capivaras (Hydrochoerus hydrochaeris, L. 1766) em Cativeiro. [Dissertação de Mestrado]. Brasil: Escola Superior de Agricultura "Luiz de Queiroz"; 1989.

13. López-Barbella S. Pubertad en hembras chigüires (Hydrochoerus hydrochoeris), Rev Fac Agron UCV 1993; 19:121-127.

14. López-Barbella S. Determinación del ciclo estral en chigüires (Hydrochoerus hydrochaeris). Acta Cient Venez 1982; 33:497-501.

15. West G, Heard D, Caulkett N. Zoo Animal and Wildlife Immobilization and Anesthesia. 1a. Edic. Iowa, USA: Blackwell Publishing; 2007.

16. Image ]. [en línea][Fecha de acceso 25 de Abril 2011] URL disponible en http:// rsbweb.nih.gov/ij/index.html

17. Cruz AA, Cardona DM, Forero MG. Prototipo de sistema experto para la clasificación morfológica de células espermáticas en bovinos. En: II Congreso Colombiano de Computación. Colombia. Bogotá: Pontificia Universidad Javeriana; 2007.

18. Escalera A. Visión por Computador, fundamentos y métodos, Madrid: Pearson Educación; 2001.

19. Souza ALP, Castelo TS, Queiroz JPAF, Barros IO, Paula VV, Oliveira MF, Silva AR. Evaluation of anesthetic protocol for the collection of semen from captive collared peccaries (Tayassu tajacu) by electroejaculation. Anim Reprod Sci 2009; 116:370-375.

20. Mollineau W, Adogwa A, Garcia G. A preliminary technique for electroejaculation of agouti (Dasyprocta leporina). Anim Reprod Sci 2007; 108:92-97.
21. Scott JV, Dziuk PJ. Evaluation of the electroejaculation technique and spermatozoa thus obtained from rats, mice and guinea pigs. Anat Rec 1959; 4:655- 64.

22. Tecirlioglu RT, Hayes ES, Trounson AO. Semen collection from mice: electroejaculation. Reprod Fertil 2002;14:363-371.

23. Busso JM, Ponzio MF, Chiaravigilo M, Fiol de cuneo M, Ruiz RD. Electroejaculation in the Chinchilla (Chinchilla lanigera): effects of anesthesia on seminal characteristics. Res Vet Sci 2005; 78:93-97.

24. Ferraz MS, de Menezes DJA, Pessoa GT, Cabral RM, Illera MJ, Silva AR, Carvalho MAM. Collection and evaluation of epididymal sperm in captive agoutis (Dasyprocta aguti). Theriogenology 2011; 75:459-462.

25. Paula TAR. Avaliação histológica e funcional do testículo de capivaras adultas (Hydrochoerus.hydrochaeris). [Tese de doutorado]. Belo Horizonte-MG: Universidade Federal de Minas Gerais. Instituto de Ciências Biológicas; 1999.

26. Moreira JR, Macdonald DW, Clarke JW. The testis of capybaras (Hydrochoerus hydrochaeris). J Mammal 1997; 78:1096-1100.

27. Dorado J, Molina I, Muñoz-Serrano A, Hidalgo $M$. Identification of sperm subpopulations with defined motility characteristics in ejaculates from Florida goats. Theriogenology 2010; 74:795-804.

28. Quintero-Moreno A, González-Villalobos D, López-Brea JJ, Esteso MC, FernándezSantos MR, Carvalho-Crociata JL, et al. Valoración morfométrica de la cabeza del espermatozoide del cerdo doméstico según su edad. Rev Cient (Maracaibo). 2009; 19(2):153-158.

29. Gage MJG. Mammalian sperm morphometry. Proc Biol Sci 1998; 265(1391):97-103. 